\title{
Double-blind randomized study of oral glutamine on the management of radio/chemotherapy-induced mucositis and dermatitis in head and neck cancer
}

\author{
DAVID LOPEZ-VAQUERO ${ }^{1}$, LUCIA GUTIERREZ-BAYARD ${ }^{2}$, JUAN-ANDRES RODRIGUEZ-RUIZ ${ }^{3}$, \\ MONICA SALDAÑA-VALDERAS ${ }^{4}$ and PEDRO INFANTE-COSSIO ${ }^{5}$
}

${ }^{1}$ Department of Oral and Maxillofacial Surgery, Donostia University Hospital, 20014 San Sebastian; Departments
of ${ }^{2}$ Radiation Oncology, ${ }^{3}$ Oral and Maxillofacial Surgery and ${ }^{4}$ Clinical Pharmacology, Puerta del Mar University
Hospital, 11009 Cadiz; ${ }^{5}$ Department of Surgery, School of Medicine, University of Seville, 41009 Seville, Spain

Received November 22, 2016; Accepted March 30, 2017

DOI: $10.3892 / \mathrm{mco} .2017 .1238$

\begin{abstract}
This randomized clinical trial was designed to determine whether glutamine administration was effective in reducing the incidence and severity of mucositis and dermatitis induced by radiotherapy (RT) or chemoradiotherapy (CHRT) in patients with head and neck cancer (HNC). Fifty patients were randomized to receive orally either L-Glutamine or placebo (25 patients in each arm). In the glutamine-treated group, $10 \mathrm{~g}$ of oral glutamine was administered three times daily. The primary endpoint was to compare the appearance of clinical mucositis between groups at the 6th week, according to the Common Terminology Criteria for Adverse Events. Secondary endpoints were: Functional mucositis, mucositis onset, cervicofacial dermatitis, pain, weight loss and assessment of quality of life (according to the M.D. Anderson Symptom Inventory-Head and Neck). In total, 76 and $87.5 \%$ developed clinical mucositis in the glutamine and placebo group, respectively. The incidence and severity grade of mucositis at the 6th week did not exhibit statistically significantly differences between the two groups, although it had a higher value in the placebo group. Significant reduction of dermatitis incidence $(\mathrm{P}=0.038)$ and severity $(\mathrm{P}=0.032)$ was found in the glutamine group. There were no differences in other outcomes such as pain, weight loss and mucositis onset, in treatment parameters including concomitant chemotherapy, radiation dose and previous surgery, or in quality of life. The present study revealed that glutamine provided slight clinical effects compared with placebo in terms of reducing oral mucositis induced by RT or CHRT in patients with HNC at the 6th week; however, the results were not statistically significant. Although
\end{abstract}

Correspondence to: Professor Pedro Infante-Cossio, Department of Surgery, School of Medicine, University of Seville, Dr Fedriani Avenue, 41009 Seville, Spain

E-mail:pinfante@us.es

Key words: glutamine, head and neck cancer, radiation therapy, chemoradiotherapy, mucositis, dermatitis the findings suggested a significant benefit in reducing the incidence and severity of dermatitis, further confirmatory studies are required.

\section{Introduction}

Radiation therapy (RT) and concomitant chemoradiotherapy (CHRT) in patients with head and neck cancer (HNC) are commonly associated with complications such as mucositis and dermatitis in the oral cavity and on the cervicofacial area. Clinically, these adverse effects are able to cause severe pain and odinophagia, increase the risk of infections, skin wounds and inflammatory ulcers, limit deglutition, cause malnutrition and negatively impact the diet and overall quality of life of patients (1). The incidence rate of mucositis varies between 85 and $100 \%$, depending on the cancer treatment regimen, with altered fractionated RT, CHRT or conventional RT $(2,3)$, whereas the incidence of acute dermatitis varies between 7 and $25 \%(4,5)$, reaching up to $49 \%$ in patients receiving RT in combination with cetuximab (6).

Numerous efforts have been made to identify effective agents for preventing and treating mucositis and dermatitis induced by RT or CHRT. Although some standard guidelines for management are available $(7,8)$, reliable and effective treatments are lacking (9). For mucositis, intensive oral care protocols, antimicrobial agents, anti-inflammatory agents, cytoprotective agents, recombinant keratinocyte growth factor-1, nutritional supplements, biostimulant agents or natural and homeopathic agents have been described (10-13). On the other hand, several topical or systemic formulations have been tried for dermatitis, all of them with ineffective results $(5,6)$. The ultimate goal of these treatments is to allow patients to receive complete radiation doses while improving their quality of life, as well as avoiding unplanned discontinuation of treatments with a subsequent negative impact on the final outcome.

Glutamine is a free primary amino acid precursor for protein synthesis, involved in cell replication for rapid cell turnover, primarily in the gastrointestinal mucosa and immune system. In circumstances of intense catabolism and stress, this organic molecule becomes indispensable as its production 
demand exceeds the capacity of endogenous synthesis (14). In addition, patients with $\mathrm{HNC}$ often have a glutamine deficit accentuated by the side effects of RT or CHRT (15).

Since the first study by Skubitz and Anderson (16) in 1996 on the role of glutamine for the prevention and treatment of oral mucositis in cancer treatments, a number of studies have demonstrated that supplementation of this amino acid may reduce the incidence and severity of mucositis during treatment by reversing cellular damage and improving cellular recovery (17). Certain studies with animal models indicated that glutamine supplementation was safe and reduced cytotoxicity-induced mucositis $(18,19)$. However, others have found no conclusive outcomes, and pointed out that more studies with higher consistency and methodological validity were needed to find more solid evidence (7). The aim of this prospective study was to determine whether oral glutamine administration was effective in reducing the incidence and severity of oral mucositis and cervicofacial dermatitis induced by RT or CHRT in patients with HNC.

\section{Patients and methods}

Patients. Patients in this trial were recruited from the Puerta del Mar University Hospital (Cadiz, Spain) between July 2010 and June 2012. The following inclusion criteria were applied: Patients with primary cancer in any head and neck location with a proven malignant biopsy, undergoing RT with or without concomitant CHRT, and 0 and I performance status grade according to ECOG (Eastern Cooperative Oncology Group Performance Status). The exclusion criteria were: Patients with a previous history of receiving RT, uncontrolled systemic or disseminated disease, presence of synchronous double malignant tumor, hypersensitivity or allergy to any of the components included in the study, uncontrolled diabetes, severe kidney or liver failure, skin diseases or autoimmune diseases.

Study design. The current study was a phase II randomized double-blind controlled study. The eligible participants were randomized into a control group or an experimental group to receive daily administration of oral glutamine or placebo for assessment of its efficacy in the management of mucositis and dermatitis following RT or concomitant CHRT. A randomization in 5 blocks of 10 patients with 1-to-1 assignment to groups was computer-generated by a statistician who was not working with the patients. These allocations were placed in sealed masked envelopes with a specific number group or an experimental group to receive a daily administration of oral glutamine or placebo. The study protocol was approved by the Ethics Committee of the Puerta del Mar University Hospital, Cadiz, Spain and by the Spanish Agency for Drugs and Health Products (number of trial registry 2009-018103-40). All patients gave written informed consent to participate.

According to the institutional protocol, the total radiation dose was fixed to $70 \mathrm{~Gy}$ in 35 fractions of $2 \mathrm{~Gy}$, or $66 \mathrm{~Gy}$ in 30-33 fractions of $2 \mathrm{~Gy}$ in postoperative RT. Depending on the case, patients received cisplatin $\left(100 \mathrm{mg} / \mathrm{m}^{2}\right)$ or cetuximab $\left(400 \mathrm{mg} / \mathrm{m}^{2}\right)$ based on renal function and the presence of systemic complications. During the study, each patient received three daily bags to be dissolved in a glass of water (orally, distributed in the three meals), containing either $10 \mathrm{~g}$ of maltodextrin as the placebo (control group) or $10 \mathrm{~g}$ of L-Glutamine as the treatment (experimental group). Both supplements were prepared by Nutricion Medica S.L. Laboratories (Madrid, Spain) in powder form packaged in single dosage pouches indistinguishable from each other, thus ensuring double-blind masking. The patients were evaluated by the same observer at the 3rd and 6th weeks during the treatment protocol, and at the 1st and 6th months post-treatment. All patients had completed dental and oral examination prior to treatment, and underwent oral care. For symptomatic mucositis, oral paracetamol tablets $500 \mathrm{mg}$ or tramadol $100 \mathrm{mg}$ were administered according to the severity of pain. The need for painkillers, adverse events associated with the study drugs and patient non-adherence to treatment were recorded.

Endpoints and measures. The primary endpoint was the appearance of clinical oral mucositis at the 6th week after treatment. Secondary endpoints included evaluation of functional mucositis, onset of mucositis, cervicofacial dermatitis, pain and weight loss. Oral mucositis and cervicofacial dermatitis were assessed according to the Common Terminology Criteria for Adverse Events version 3.0 (CTCAE v3.0). Clinical mucositis was graded from grade 0 (no mucositis) to grade 4 (symptomatic associated with life threatening consequences, tissue necrosis, significant spontaneous bleeding). Functional mucositis was evaluated from grade 0 (no mucositis) to grade 4 (symptoms associated with life-threatening consequences). Dermatitis on the cervicofacial area was assessed from grade 1 (faint erythema or dry desquamation) to grade 4 (skin necrosis or ulceration of full thickness of dermis; spontaneous bleeding from involved site). Data regarding pain were collected using a visual analog scale (VAS) from 0 ('no pain') to 10 ('insupportable pain'). Evaluation of treatment tolerance was based on occurrence of adverse events during the trial. Patients fulfilled the quality of life questionnaire of the M.D. Anderson Symptom Inventory-Head and Neck (MDASI-HN), comprising 3 subscales: 13 items that rated the severity of general symptoms associated with cancer, $9 \mathrm{HNC}$-specific items that rated the severity of symptoms particularly associated with HNC and 6 items that assessed how severely symptoms interfered with daily activities. The core and HNC-specific symptoms were rated on a 0-10 scale to indicate the presence and severity of the symptom, with 0 indicating 'not present' and 10 indicating 'as bad as you can imagine.'

Statistical analysis. The sample size was calculated according to an expected clinical mucositis appearance of $100 \%$ at the 6th month. Expecting to find a reduction of $75 \%$ after prescribing glutamine ( $\delta$ value of $25 \%$ ), a $5 \% \alpha$ error and an $80 \% \beta$ value (study power) were selected, assuming a 5\% loss. With these data, the necessary number of participants to obtain statistical significance was 50 patients ( 25 per group). Intention to treat and per protocol analyses were performed on the population.

The results were analyzed using the SPSS version 15.0 (IBM Corp., Chicago, IL, USA). All statistical tests achieved a significance level of $\mathrm{P}<0.05$ for a bilateral significance. The baseline characteristics of groups were compared using analysis of variance for continuous variables. In the case of discrete variables, the distribution of absolute frequencies and 
percentages were obtained. Discrete variables were compared using the $\chi^{2}$ statistic, and continuous variables using the Student's t-test. Comparisons between the two groups were performed using analysis of covariance. The results of VAS scores were presented as the mean, median and standard deviation.

\section{Results}

Fifty patients between 32 and 79 years of age (mean, 60.24) were included in the intention to treat analysis, $78 \%$ were male and $22 \%$ were female. One female dropped out due to RT suspension. As a result, 24 patients in the control group and 25 patients in the experimental group were included in the full per protocol analysis (Fig. 1). On conducting the statistical analysis, the intention to treat analysis and per protocol analysis produced identical results for all criterion measures; therefore, only the per protocol analysis was used to describe the results.

The distribution of patients according to baseline patient characteristics, diagnostic and treatment parameters were similar between the study groups (Table I). In total, $45 \%$ of patients received $\mathrm{RT}$ and $55 \%$ concomitant CHRT. The dose of radiation was $70 \mathrm{~Gy}$ in $91.8 \%$ of patients and $66 \mathrm{~Gy}$ in $8.2 \%$. Cisplatin and cetuximab was indicated in 13 and 14 patients, respectively; $63.3 \%$ of patients had previously underwent surgery.

The incidence of clinical mucositis was $87.5 \%$ in the placebo group and $76 \%$ in the glutamine group (81.6\% of global incidence) (Table II). The incidence and severity grade of clinical and functional mucositis at the 6th week did not exhibit statistically significantly differences between the two groups. The comparison of clinical and functional mucositis had a higher value in placebo group, although without statistical difference. A direct significant statistical correlation was found between the values of the clinical and functional mucositis $(\mathrm{P}=0.01)$, with a coefficient of 0.71 and 0.597 at the $3 \mathrm{rd}$ and 6 th week, respectively.

A statistically significant reduction of the incidence $(\mathrm{P}=0.038)$ and severity $(\mathrm{P}=0.032)$ of dermatitis was found in the glutamine group at the 6 th week. There were no differences between groups in the mucositis appearance according to any of treatment parameters (concomitant chemotherapy, radiation dose and previous surgery). No statistically significant differences between the two groups were identified for pain, weight loss and mucositis onset (Table III). The analysis of the quality of life questionnaire (MDASI-HN) revealed no significant differences between groups in any items at the 6th week. No patient discontinued the study medication due to adverse effects.

\section{Discussion}

The effectiveness of glutamine for the prevention and treatment of oral mucositis induced by RT has been examined recently in some meta-analyses. One meta-analysis (20) identified 5 clinical randomized controlled trials that included 234 patients with HNC. The conclusions of this meta-analysis revealed that glutamine significantly reduced the risk and severity of oral mucositis induced by RT or CHRT compared with either placebo or no treatment (risk ratio 0.17;

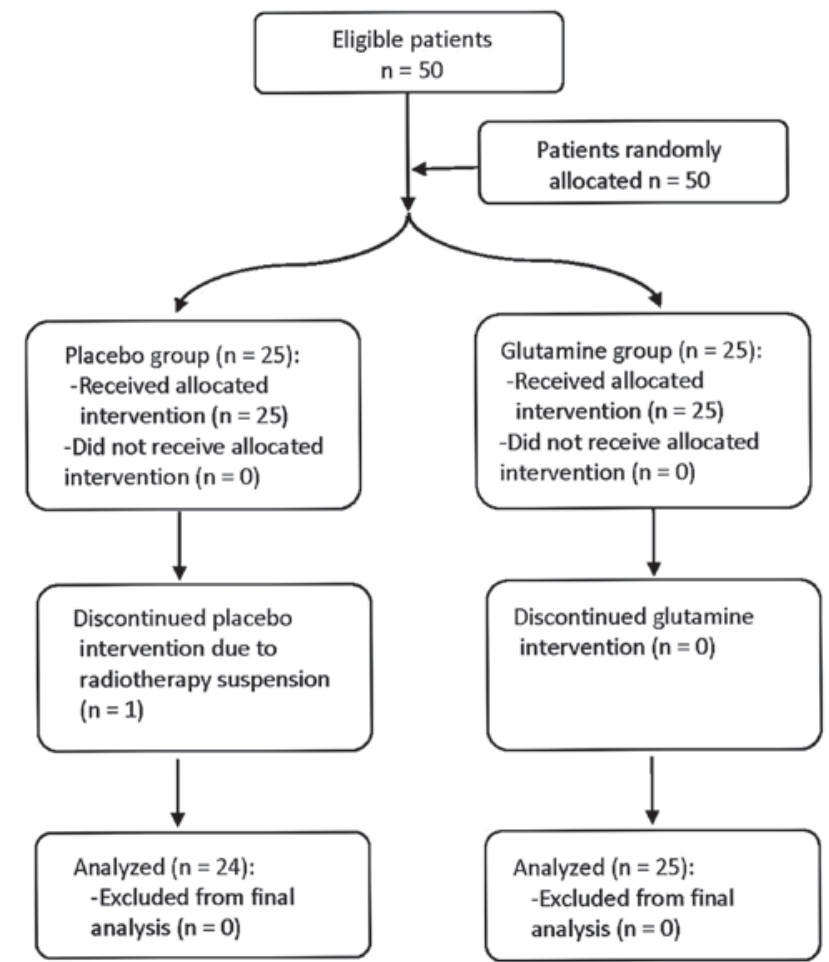

Figure 1. Flow diagram of the patient treatment process.

95\% CI 0.06-0.47) (21-25). Another systematic review of well-designed studies in various solid tumors and patients with hematological cancer revealed inadequate or conflicting evidence (26), as five studies did not find glutamine to be effective (27-30), while four studies did (19,31-33), suggesting a requirement for further studies on the use of oral glutamine to guide clinicians on which interventions are truly effective.

The findings of the present study did not demonstrate the primary study endpoint in a statistically significant manner. However, the incidence and severity of clinical and functional mucositis tended to be clinically lower in the glutamine group in comparison with the experimental group, although it did not reach statistical significance. The frequency of clinical mucositis was of $87.5 \%$ for the placebo group vs. $76 \%$ for the glutamine group and, of a total of 9 patients who did not show mucositis, 6 were found in the glutamine group. None of patients presented with a severity grade of 4 at the 6th week, revealing a homogeneous distribution of clinical mucositis between the two groups.

Although the present study was unable to demonstrate the effectiveness of the primary endpoint, oral glutamine significantly reduced the incidence and severity of dermatitis produced in the radiation fields, as a secondary endpoint. A previous study reported that $\beta$-hydroxy- $\beta$-methylbutyrate/arginine/glutamine supplementation was potentially effective in the prevention of radiation dermatitis in patients with $\mathrm{HNC}$, as this supplementation was a protective nutrient with anti-inflammatory effects favoring the healing of inflammatory skin wounds (34).

In the current study, there were no differences in other outcomes including pain, weight loss and mucositis onset, and in treatment parameters such as concomitant chemotherapy, radiation dose and previous surgery. This study was not able to 
Table I. Baseline patient characteristics and diagnostic parameters.

\begin{tabular}{|c|c|c|c|c|c|}
\hline & Placebo group $(\mathrm{n}=25)$ & $\%$ & Glutamine group $(\mathrm{n}=25)$ & $\%$ & P-value \\
\hline Sex & & & & & 0.496 \\
\hline Male & 20 & 80 & 18 & 72 & \\
\hline Female & 5 & 20 & 7 & 28 & \\
\hline Median age, years & $61.5(32-81)$ & & 59. (39-78) & & 0.432 \\
\hline Tobacco use & & & & & 0.358 \\
\hline No smoker & 6 & 24 & 7 & 28 & \\
\hline$<20$ cigarettes & 5 & 20 & 1 & 4 & \\
\hline 20-40 cigarettes & 6 & 24 & 7 & 28 & \\
\hline$>40$ cigarettes & 2 & 8 & 5 & 20 & \\
\hline Ex-smoker & 5 & 20 & 5 & 20 & \\
\hline Alcohol & & & & & 0.368 \\
\hline No & 11 & 44 & 10 & 40 & \\
\hline Yes & 12 & 48 & 11 & 44 & \\
\hline Ex-alcoholic & 2 & 8 & 4 & 16 & \\
\hline Median weight, $\mathrm{kg}$ & $76.7(52.6-102.8)$ & & $70.8(37.6-96)$ & & \\
\hline Median pain & $0.49(0-8)$ & & $0.61(0-7)$ & & \\
\hline Primary tumor site & & & & & 0.621 \\
\hline Oral cavity & 9 & 36 & 8 & 32 & \\
\hline Oropharynx & 5 & 20 & 6 & 24 & \\
\hline Nasopharynx & 1 & 4 & 1 & 4 & \\
\hline Hypopharynx & 2 & 8 & 1 & 4 & \\
\hline Larynx & 8 & 32 & 9 & 36 & \\
\hline Tumor histopathology & & & & & 0.594 \\
\hline Squamous cell carcinoma & 21 & 84 & 22 & 88 & \\
\hline Adenocarcinoma & 2 & 8 & 1 & 4 & \\
\hline Mucoepidermoid & 1 & 4 & 2 & 8 & \\
\hline Adenoid cystic carcinoma & 1 & 4 & 0 & 0 & \\
\hline AJCC stage & & & & & 0.71 \\
\hline I & 0 & 0 & 0 & 0 & \\
\hline II & 5 & 20 & 4 & 16 & \\
\hline III & 7 & 28 & 5 & 20 & \\
\hline IV & 13 & 52 & 16 & 64 & \\
\hline ECOG-PS & & & & & 0.98 \\
\hline 0 & 24 & 96 & 25 & 100 & \\
\hline 1 & 1 & 4 & 0 & 0 & \\
\hline
\end{tabular}

AJCC, American Joint Committee on Cancer; ECOG-PS, Eastern Cooperative Oncology Group Performance Status.

demonstrate an improvement in the quality of life of patients as no statistical differences were also found between the two groups in the assessment of cancer-associated symptoms with the MDASI-HN quality of life questionnaire.

Very few clinical trials on oral glutamine administration for the prevention of oral mucositis in patients with HNC have been reported (21-24). In general terms, the majority of studies have described favorable results concerning oral glutamine administration, and consequently the findings of the present study are not fully consistent with the indexed literature. However, the comparison with these previous studies is challenging, as the choice of the primary endpoint, the $\delta$ value and the magnitude of the clinically relevant difference, were not revealed in the previous studies. In the present study, mucositis and dermatitis were considered as qualitative variables, which gave more verisimilitude to clinical reality unlike other studies in which they were classified as quantitative, thus losing much data analysis.

The results of the present study must be interpreted with caution due to the short follow-up period and the reduced study sample size. The sample size depended upon the $25 \%$ $\delta$ value, estimated based on the assumption that the placebo group should have a $100 \%$ incidence of clinical mucositis at the 6th week, which did not occur. Consequently, a lower 
Table II. Incidence and severity of mucositis and dermatitis.

\begin{tabular}{|c|c|c|c|c|c|}
\hline & Placebo group $(n=24)$ & $\%$ & Glutamine group $(n=25)$ & $\%$ & P-value \\
\hline Incidence of clinical mucositis & 21 & 87.5 & 19 & 76 & 0.324 \\
\hline Grade of clinical mucositis & & & & & 0.341 \\
\hline Grade 0 & 3 & 12.5 & 6 & 24 & \\
\hline Grade 1 & 10 & 41.7 & 8 & 32 & \\
\hline Grade 2 & 9 & 36.4 & 10 & 40 & \\
\hline Grade 3 & 2 & 8.4 & 1 & 4 & \\
\hline Grade 4 & 0 & 0 & 0 & 0 & \\
\hline Incidence of functional mucositis & 18 & 75 & 19 & 76 & 0.511 \\
\hline Grade of functional mucositis & & & & & 0.198 \\
\hline Grade 0 & 6 & 25 & 6 & 24 & \\
\hline Grade 1 & 8 & 33.3 & 9 & 36 & \\
\hline Grade 2 & 10 & 41.7 & 7 & 28 & \\
\hline Grade 3 & 0 & 0 & 3 & 12 & \\
\hline Grade 4 & 0 & 0 & 0 & 0 & \\
\hline Incidence of dermatitis & 24 & 100 & 21 & 84 & $0.038^{\mathrm{a}}$ \\
\hline Grade of dermatitis & & & & & $0.032^{\mathrm{a}}$ \\
\hline Grade 0 & 0 & 0 & 4 & 16 & \\
\hline Grade 1 & 11 & 45.8 & 15 & 60 & \\
\hline Grade 2 & 12 & 50 & 5 & 20 & \\
\hline Grade 3 & 1 & 4.2 & 1 & 4 & \\
\hline Grade 4 & 0 & 0 & 0 & 0 & \\
\hline
\end{tabular}

${ }^{\mathrm{a}} \mathrm{P}<0.05$.

Table III. Effect of glutamine on several outcomes.

\begin{tabular}{|c|c|c|c|c|}
\hline Variable & Units & Placebo group $(n=24)$ & Glutamine group $(n=25)$ & P-value \\
\hline Pain & VAS (0-10) & 1.96 & 2.32 & 0.574 \\
\hline Weight & $\mathrm{Kg}$ & -2.55 & -3.3 & 0.526 \\
\hline Onset of mucositis & Days & 29.91 & 28.38 & 0.726 \\
\hline \multicolumn{5}{|c|}{ MDASI-HN questionnaire } \\
\hline & General items & 2.23 & 1.7 & 0.374 \\
\hline & Specific items & 2.6 & 2.6 & 0.48 \\
\hline & Impact on daily activities & 3.05 & 2.58 & 0.54 \\
\hline & Global & 2.34 & 1.85 & 0.222 \\
\hline
\end{tabular}

MDASI-H, M. D. Anderson Symptom Inventory-Head and Neck; VAS, visual analog scale.

$\delta$ value may have favored a larger sample size. This study was a single center study performed in the authors' setting. Although the distribution of patients was similar between groups and treatment parameters such as chemotherapy, radiation dose or previous surgery showed no difference between groups, a subgroup of analysis could have been performed if the number of subjects were not too low. Consequently, multicenter and large-scale studies are warranted. The strengths of this study were based on its randomized double-blind placebo-controlled study design. Throughout the trial, the main researcher monitored the patient test adherence, which resulted in a $100 \%$ adherence, and also performed a count of bags used and surplus.

Therefore, it is only possible to conclude that, in this double-blind, randomized study, oral glutamine provided slight clinical effects compared with placebo in reducing oral mucositis induced by RT or CHRT in patients with HNC at the 6th week, although the results were not statically significant. While the findings of the study suggested a significant benefit in reducing the incidence and severity of dermatitis, further confirmatory studies with a new primary endpoint and a larger sample size are required. 


\section{Acknowledgements}

The present study formed part of a thesis and L-glutamine and oral nutrition supplements were gifts of Nutrition Medica S.L. Laboratories (Madrid, Spain).

\section{References}

1. Moslemi D, Nokhandani AM, Otaghsaraei MT, Moghadamnia Y, Kazemi S and Moghadamnia AA: Management of chemo/radiation-induced oral mucositis in patients with head and neck cancer: A review of the current literature. Radiother Oncol 120: 13-20, 2016.

2. Trotti A, Bellm LA, Epstein JB, Frame D, Fuchs HJ, Gwede CK, Komaroff E, Nalysnyk L and Zilberberg MD: Mucositis incidence, severity and associated outcomes in patients with head and neck cancer receiving radiotherapy with or without chemotherapy: A systematic literature review. Radiother Oncol 66 : 253-262, 2003.

3. Harada K, Ferdous T, Horinaga D, Uchida K, Mano T, Mishima K, Park S, Hanazawa H, Takahashi S, Okita A, et al: Efficacy of elemental diet on prevention for chemoradiotherapy-induced ora mucositis in patients with oral squamous cell carcinoma. Support Care Cancer 24: 953-959, 2016.

4. Cooper JS, Pajak TF, Forastiere AA, Jacobs J, Campbell BH, Saxman SB, Kish JA, Kim HE, Cmelak AJ, Rotman M, et al: Postoperative concurrent radiotherapy and chemotherapy for high-risk squamous-cell carcinoma of the head and neck. N Engl J Med 350: 1937-1944, 2004.

5. Elliott EA, Wright JR, Swann RS, Nguyen-Tân F, Takita C, Bucci MK, Garden AS, Kim H, Hug EB, Ryu J, et al: Phase III trial of an emulsion containing trolamine for the prevention of radiation dermatitis in patients with advanced squamous cell carcinoma of the head and neck: Results of radiation therapy oncology group trial 99-13. J Clin Oncol 24: 2092-2097, 2006.

6. Giro C, Berger B, Bölke E, Ciernik IF, Duprez F, Locati L, Maillard S, Ozsahin M, Pfeffer R, Robertson AG, et al: High rate of severe radiation dermatitis during radiation therapy with concurrent cetuximab in head and neck cancer: Results of a survey in EORTC institutes. Radiother Oncol 90: 166-171, 2009.

7. Mead GM: Management of oral mucositis associated with cancer chemotherapy. Lancet 359: 815-816, 2002.

8. Vélez I, Tamara LA and Mintz S: Management of oral mucositis induced by chemotherapy and radiotherapy: An update. Quintessence Int 35: 129-136, 2004.

9. Lalla RV, Bowen J, Barasch A, Elting L, Epstein J, Keefe DM, McGuire DB, Migliorati C, Nicolatou-Galitis O, Peterson DE, et al: MASCC/ISOO clinical practice guidelines for the management of mucositis secondary to cancer therapy. Cancer 120: 1453-1461, 2014.

10. Stokman MA, Spijkervet FK, Boezen HM, Schouten JP, Roodenburg JL and de Vries EG: Preventive intervention possibilities in radiotherapy- and chemotherapy-induced oral mucositis: Results of meta-analyses. J Dent Res 85: 690-700, 2006.

11. Keefe DM, Schubert MM, Elting LS, Sonis ST, Epstein JB, Raber-Durlacher JE, Migliorati CA, McGuire DB, Hutchins RD and Peterson DE; Mucositis Study Section of the Multinational Association of Supportive Care in Cancer and the International Society for Oral Oncology: Updated clinical practice guidelines for the prevention and treatment of mucositis. Cancer 109 820-831, 2007.

12. Henke M, Alfonsi M, Foa P, Giralt J, Bardet E, Cerezo L, Salzwimmer M, Lizambri R, Emmerson L, Chen MG and Berger D: Palifermin decreases severe oral mucositis of patients undergoing postoperative radiochemotherapy for head and neck cancer: A randomized, placebo-controlled trial. J Clin Oncol 29: 2815-2820, 2011

13. Xu JL, Xia R, Sun ZH, Sun L, Min X, Liu C, Zhang H and Zhu YM: Effects of honey use on the management of radio/chemotherapy-induced mucositis: A meta-analysis of randomized controlled trials. Int J Oral Maxillofac Surg 45: $1618-1625,2016$

14. Wischmeyer PE: Clinical applications of L-glutamine: Past, present, and future. Nutr Clin Pract 18: 377-385, 2003.

15. Neu J, DeMarco V and Li N: Glutamine: Clinical applications and mechanisms of action. Curr Opin Clin Nutr Metab Care 5: 69-75, 2002
16. Skubitz KM and Anderson PM: Oral glutamine to prevent chemotherapy induced stomatitis: A pilot study. J Lab Clin Med 127: 223-228, 1996.

17. Silverman S Jr: Diagnosis and management of oral mucositis. J Support Oncol 5 (2 Suppl 1): S13-S21, 2007.

18. Savarese DM, Savy G, Vahdat L, Wischmeyer PE and Corey B: Prevention of chemotherapy and radiation toxicity with glutamine. Cancer Treat Rev 29: 501-513, 2003.

19. Choi K, Lee SS, Oh SJ, Lim SY, Lim SY, Jeon WK, Oh TY and Kim JW: The effect of oral glutamine on 5-fluorouracil/leucovorin-induced mucositis/stomatitis assessed by intestinal permeability test. Clin Nutr 26: 57-62, 2007.

20. Leung HW and Chan AL: Glutamine in alleviation of radiation-induced severe oral mucositis: A meta-analysis. Nutr Cancer 68: 734-742, 2016.

21. Huang EY, Leung SW, Wang CJ, Chen HC, Sun LM, Fang FM, Yeh SA, Hsu HC and Hsiung CY: Oral glutamine to alleviate radiation-induced oral mucositis: A pilot randomized trial. Int J Radiat Oncol Biol Phys 46: 535-539, 2000.

22. Cerchietti LC, Navigante AH, Lutteral MA, Castro MA, Kirchuk R, Bonomi M, Cabalar ME, Roth B, Negretti G, Sheinker B and Uchima P: Double-blinded, placebo-controlled trial on intravenous L-alanyl-L-glutamine in the incidence of oral mucositis following chemoradiotherapy in patients with head and-neck cancer. Int J Radiat Oncol Biol Phys 65: 1330-1337, 2006.

23. Chattopadhyay S, Saha A, Azam M, Mukherjee A and Sur PK: Role of oral glutamine in alleviation and prevention of radiation-induced oral mucositis: A prospective randomized study. South Asian J Cancer 3: 8-12, 2014.

24. Tsujimoto T, Yamamoto Y, Wasa M, Takenaka Y, Nakahara S, Takagi T, Tsugane M, Hayashi N, Maeda K, Inohara H, et al: L-glutamine decreases the severity of mucositis induced by chemoradiotherapy in patients with locally advanced head and neck cancer: A double-blind, randomized, placebo-controlled trial. Oncol Rep 33: 33-39, 2015.

25. Vidal-Casariego A, Calleja-Fernández A, Ballesteros-Pomar MD and Cano-Rodríguez I: Efficacy of glutamine in the prevention of oral mucositis and acute radiation induced esophagitis: A retrospective study. Nutr Cancer 65: 424-429, 2013.

26. Yarom N, Ariyawardana A, Hovan A, Barasch A, Jarvis V, Jensen SB, Zadik Y, Elad S, Bowen J and Lalla RV; Mucositis Study Group of the Multinational Association of Supportive Care in Cancer/International Society of Oral Oncology (MASCC/ISOO): Systematic review of natural agents for the management of oral mucositis in cancer patients. Support Care Cancer 21: 3209-3221, 2013.

27. Jebb SA, Osborne RJ, Maughan TS, Mohideen N, Mack P, Mort D, Shelley MD and Elia M: 5-fluorouracil and folinic acid induced mucositis: No effect of oral glutamine supplementation. Br J Cancer 70: 732-735, 1994.

28. Anderson PM, Schroeder G and Skubitz KM: Oral glutamine reduces the duration and severity of stomatitis after cytotoxic cancer chemotherapy. Cancer 83: 1433-1439, 1998.

29. Okuno SH, Woodhouse CO, Loprinzi CL, Sloan JA, LaVasseur BI, Clemens-Schutjer D, Swan D, Axvig C, Ebbert LP, Tirona MR, et al: Phase III controlled evaluation of glutamine for decreasing stomatitis in patients receiving fluorouracil (5-FU)-based chemotherapy. Am J Clin Oncol 22: 258-261, 1999.

30. Ward E, Smith M, Henderson M, Reid U, Lewis I, Kinsey S, Allgar V, Bowers D and Picton SV: The effect of high-dose enteral glutamine on the incidence and severity of mucositis in paediatric oncology patients. Eur J Clin Nutr 63: 134-140, 2009.

31. Rubio IT, Cao Y, Hutchins LF, Westbrook KC and Klimberg VS: Effect of glutamine on methotrexate efficacy and toxicity. Ann Surg 227: 772-778; 778-780, 1998.

32. Cockerham MB, Weinberger BB and Lerchie SB: Oral glutamine for the prevention of oral mucositis associated with high-dose paclitaxel and melphalan for autologous bone marrow transplantation. Ann Pharmacother 34: 300-303, 2000.

33. Peterson DE, Jones JB and Petit RG II: Randomized, placebo-controlled trial of Saforis for prevention and treatment of oral mucositis in breast cancer patients receiving anthracycline-based chemotherapy. Cancer 109: 322-331, 2007.

34. Imai T, Matsuura K, Asada Y, Sagai S, Katagiri K, Ishida E, Saito D, Sadayasu R, Wada H and Saijo S: Effect of HMB/Arg/Gln on the prevention of radiation dermatitis in head and neck cancer patients treated with concurrent chemoradiotherapy. Jpn J Clin Oncol 44: 422-427, 2014. 